Article

\title{
Some Quantum Estimates of Hermite-Hadamard Inequalities for Quasi-Convex Functions
}

\author{
Hefeng Zhuang ${ }^{1,+}$, Wenjun Liu ${ }^{1, *,+}$ and Jaekeun Park ${ }^{2}$ \\ 1 College of Mathematics and Statistics, Nanjing University of Information Science and Technology, \\ Nanjing 210044, China; hfzhuang11@163.com \\ 2 Department of Mathematics, Hanseo University, Chungnam-do, Seosan-si 356-706, Korea; \\ jkpark@hanseo.ac.kr \\ * Correspondence: wjliu@nuist.edu.cn; Tel.: +86-25-5873-1160 \\ + These authors contributed equally to this work.
}

Received: 18 December 2018; Accepted: 2 February 2019; Published: 5 February 2019

check for updates

\begin{abstract}
In this paper, we develop some quantum estimates of Hermite-Hadamard type inequalities for quasi-convex functions. In some special cases, these quantum estimates reduce to the known results.
\end{abstract}

Keywords: quantum estimates; Hermite-Hadamard type inequalities; quasi-convex

\section{Introduction}

\subsection{Current State of Hermite-Hadamard Inequalities}

Many important inequalities are established for the class of convex functions [1], but one of the most famous is the so-called Hermite-Hadamard inequality, which was first discovered by Hermite in 1881 , and is stated as follows: Let $f: I \subseteq \mathbb{R} \rightarrow \mathbb{R}$ be a convex function, where $a, b \in I$ with $a<b$. Then

$$
f\left(\frac{a+b}{2}\right) \leq \frac{1}{b-a} \int_{a}^{b} f(x) d x \leq \frac{f(a)+f(b)}{2} .
$$

This famous result can be considered as a necessary and sufficient condition for a function to be convex. Hermite-Hadamard's inequality has raised many scholars' attention, and a variety of refinements and generalizations have been found (see [1-20]).

In [16], Özdemir used the following lemma and established some estimates on it via quasi-convex functions.

Lemma 1. ([16], Lemma 1) Let $f: I \subset \mathbb{R} \rightarrow \mathbb{R}$ be a twice differentiable mapping on $I^{0}, a, b \in I$ with $a<b$ and $f^{\prime \prime}$ be integrable on $[a, b]$. Then the following equality holds:

$$
\frac{f(a)+f(b)}{2}-\frac{1}{b-a} \int_{a}^{b} f(x) d x=\frac{(b-a)^{2}}{2} \int_{0}^{1} s(1-s) f^{\prime \prime}(s a+(1-s) b) d s .
$$

Theorem 1. ([16], Theorem 2) Let $f: I^{0} \subset[0, \infty) \rightarrow \mathbb{R}$ be a twice differentiable mapping on $I^{0}$, such that $f^{\prime \prime} \in L[a, b], a, b \in I$ with $a<b$. If $\left|f^{\prime \prime}\right|^{r}$ is quasi-convex on $[a, b]$ for $r \geq 1$, then the following inequality holds:

$$
\left|\frac{f(a)+f(b)}{2}-\frac{1}{b-a} \int_{a}^{b} f(x) d x\right| \leq \frac{(b-a)^{2}}{4}\left(\frac{2}{(r+1)(r+2)}\right)^{\frac{r-1}{r}}\left(\sup \left\{\left|f^{\prime \prime}(a)\right|^{r},\left|f^{\prime \prime}(b)\right|^{r}\right\}\right)^{\frac{1}{r}} \text {. }
$$


Theorem 2. ([16], Theorem 3) Let $f: I^{0} \subset[0, \infty) \rightarrow \mathbb{R}$ be a twice differentiable mapping on $I^{0}$, such that $f^{\prime \prime} \in L[a, b], a, b \in I$ with $a<b$. If $\left|f^{\prime \prime}\right|^{r}$ is quasi-convex on $[a, b]$ for $r>1$, then the following inequality holds:

$$
\left|\frac{f(a)+f(b)}{2}-\frac{1}{b-a} \int_{a}^{b} f(x) d x\right| \leq \frac{(b-a)^{2}}{2^{1+\frac{1}{r}}}(\beta(2, p+1))^{\frac{1}{p}}\left(\sup \left\{\left|f^{\prime \prime}(a)\right|^{r},\left|f^{\prime \prime}(b)\right|^{r}\right\}\right)^{\frac{1}{r}},
$$

where $\frac{1}{p}+\frac{1}{r}=1$ and $\beta($, $)$ is Euler Beta Function:

$$
\beta(x, y)=\int_{0}^{1} t^{x-1}(1-t)^{y-1} d t, \quad x, y>0 .
$$

In [2], Alomari et al. established the following inequalities through Lemma 1.

Theorem 3. ([2], Theorem 3) Let $f: I \subset \mathbb{R} \rightarrow \mathbb{R}$ be a twice differentiable mapping on $I^{0}, a, b \in I$ with $a<b$ and $f^{\prime \prime}$ be integrable on $[a, b]$. If $\left|f^{\prime \prime}\right|$ is quasi-convex on $[a, b]$, then the following inequality holds:

$$
\left|\frac{f(a)+f(b)}{2}-\frac{1}{b-a} \int_{a}^{b} f(x) d x\right| \leq \frac{(b-a)^{2}}{12} \sup \left\{\left|f^{\prime \prime}(a)\right|,\left|f^{\prime \prime}(b)\right|\right\} .
$$

Theorem 4. ([2], Theorem 4) Let $f: I \subset \mathbb{R} \rightarrow \mathbb{R}$ be a twice differentiable mapping on $I^{0}, a, b \in I$ with $a<b$ and $f^{\prime \prime}$ be integrable on $[a, b]$. If $\left|f^{\prime \prime}\right|^{p /(p-1)}$ is quasi-convex on $[a, b]$ for $p>1$, then the following inequality holds:

$$
\left|\frac{f(a)+f(b)}{2}-\frac{1}{b-a} \int_{a}^{b} f(x) d x\right| \leq \frac{(b-a)^{2}}{8}\left(\frac{\sqrt{\pi}}{2}\right)^{\frac{1}{p}}\left(\frac{\Gamma(1+p)}{\Gamma\left(\frac{3}{2}+p\right)}\right)^{\frac{1}{p}}\left(\sup \left\{\left|f^{\prime \prime}(a)\right|^{r},\left|f^{\prime \prime}(b)\right|^{r}\right\}\right)^{\frac{1}{r}}
$$

where $r=p /(p-1)$.

Theorem 5. ([2], Theorem 5) Let $f: I \subset \mathbb{R} \rightarrow \mathbb{R}$ be a twice differentiable mapping on $I^{0}, a, b \in I$ with $a<b$ and $f^{\prime \prime}$ be integrable on $[a, b]$. If $\left|f^{\prime \prime}\right|^{r}$ is quasi-convex on $[a, b]$ for $q \geq 1$, then the following inequality holds:

$$
\left|\frac{f(a)+f(b)}{2}-\frac{1}{b-a} \int_{a}^{b} f(x) d x\right| \leq \frac{(b-a)^{2}}{12}\left(\sup \left\{\left|f^{\prime \prime}(a)\right|^{r},\left|f^{\prime \prime}(b)\right|^{r}\right\}\right)^{\frac{1}{r}} .
$$

\subsection{Motivation of Quantum Estimates}

In recent years, many researchers have shown their interest in studying and investigating quantum calculus. Quantum analysis has large applications in many mathematical areas such as number theory ([21]), special functions ([22]), quantum mechanics ([23]) and mathematical inequalities. At present, $q$-analogues of many identities and inequalities have been established $([13-15,19,20,24])$.

The Hermite-Hadamard inequality has been extended by considering its quantum estimates. For example, in [13], Noor et al. established the following lemma and developed some quantum estimates for it.

Lemma 2. ([13], Lemma 3.1) Let $f: I=[a, b] \subset \mathbb{R} \rightarrow \mathbb{R}$ be a q-differentiable function on $I^{0}$ (the interior of $I$ ) with $D_{q}$ be continuous and integrable on I where $0<q<1$, then

$$
\frac{1}{b-a} \int_{a}^{b} f(x)_{a} d_{q} x-\frac{q f(a)+f(b)}{1+q}=\frac{q(b-a)}{1+q} \int_{0}^{1}(1-(1+q) t)_{a} D_{q} f((1-t) a+t b)_{0} d_{q} t .
$$


Theorem 6. ([13], Theorem 3.2) Let $f: I=[a, b] \subset \mathbb{R} \rightarrow \mathbb{R}$ be a q-differentiable function on $I^{0}$ (the interior of I) with ${ }_{a} D_{q}$ be continuous and integrable on I where $0<q<1$. If $\left|{ }_{a} D_{q} f\right|^{r}, r \geq 1$ is a convex function, then

$$
\begin{aligned}
& \left|\frac{1}{b-a} \int_{a}^{b} f(x)_{a} d_{q} x-\frac{q f(a)+f(b)}{1+q}\right| \\
\leq & \frac{q(b-a)}{1+q}\left(\frac{2 q}{(1+q)^{2}}\right)^{1-\frac{1}{r}}\left(\left.\left.\frac{q\left(1+3 q^{2}+2 q^{3}\right)}{\left(1+q+q^{2}\right)(1+q)^{3}}\right|_{a} D_{q} f(a)\right|^{r}+\left.\left.\frac{q\left(1+4 q+q^{2}\right)}{\left(1+q+q^{2}\right)(1+q)^{3}}\right|_{a} D_{q} f(b)\right|^{r}\right)^{\frac{1}{r}} .
\end{aligned}
$$

Theorem 7. ([13], Theorem 3.3) Let $f: I=[a, b] \subset \mathbb{R} \rightarrow \mathbb{R}$ be a q-differentiable function on $I^{0}$ (the interior of I) with ${ }_{a} D_{q}$ be continuous and integrable on I where $0<q<1$. If $\left|{ }_{a} D_{q} f\right|^{r}$ is a convex function where $p, r>1, \frac{1}{p}+\frac{1}{r}=1$, then

$$
\begin{aligned}
& \left|\frac{1}{b-a} \int_{a}^{b} f(x)_{a} d_{q} x-\frac{q f(a)+f(b)}{1+q}\right| \\
\leq & \frac{q(b-a)}{1+q}\left(\frac{2 q}{(1+q)^{2}}\right)^{\frac{1}{p}}\left(\frac{q\left(1+3 q^{2}+2 q^{3}\right)}{\left(1+q+q^{2}\right)(1+q)^{3}}\left|{ }_{a} D_{q} f(a)\right|^{r}+\frac{q\left(1+4 q+q^{2}\right)}{\left(1+q+q^{2}\right)(1+q)^{3}}\left|{ }_{a} D_{q} f(b)\right|^{r}\right)^{\frac{1}{r}} .
\end{aligned}
$$

The main purpose of this paper is to use a new quantum integral identity established in [11] to develop some quantum estimates of Hermite-Hadamard type inequalities for quasi-convex functions (Section 3). These quantum estimates of Hermite-Hadamard type inequalities reduces to Theorems 1-5 as $q \rightarrow 1$.

\subsection{Possible Applications of the Estimates}

Quantum calculus has large applications in many mathematical areas. We expect these new quantum estimates for Hermite-Hadamard type inequalities to have potential applications in the fields of integral inequalities, approximation theory, special means theory, optimization theory, information theory and numerical analysis.

\section{Preliminaries}

In this section, we first recall some previously known concepts on $q$-calculus which will be used in this paper.

Let $J=[a, b] \subseteq \mathbb{R}$ be an interval and $0<q<1$ be a constant.

Definition 1. [19] Assume $f: J \rightarrow \mathbb{R}$ is a continuous function and let $x \in J$. Then q-derivative on $J$ of function $f$ at $x$ is defined as

$$
{ }_{a} D_{q} f(x)=\frac{f(x)-f(q x+(1-q) a)}{(1-q)(x-a)}, x \neq a, \quad{ }_{a} D_{q} f(a)=\lim _{x \rightarrow a} D_{q} f(x) .
$$

We say that $f$ is q-differentiable on J provided ${ }_{a} D_{q} f(x)$ exists for all $x \in J$. Note that if $a=0$ in (2.1), then ${ }_{0} D_{q} f=D_{q} f$, where $D_{q}$ is the well-known q-derivative of the function $f(x)$ defined by

$$
D_{q} f(x)=\frac{f(x)-f(q x)}{(1-q) x}
$$

Definition 2. [19] Let $f: J \rightarrow \mathbb{R}$ be a continuous function. We define the second-order q-derivative on interval $J$, which denoted as ${ }_{a} D_{q}^{2} f$, provided ${ }_{a} D_{q} f$ is q-differentiable on J with ${ }_{a} D_{q}^{2} f={ }_{a} D_{q}\left({ }_{a} D_{q} f\right): J \rightarrow \mathbb{R}$. Similarly, we define higher order $q$-derivative on $J,{ }_{a} D_{q}^{n}: J_{k} \rightarrow \mathbb{R}$. 
Definition 3. [19] Let $f: J \subset \mathbb{R} \rightarrow \mathbb{R}$ be a continuous function. Then q-integral on $J$ is defined by

$$
\int_{a}^{x} f(t){ }_{a} d_{q} t=(1-q)(x-a) \sum_{n=0}^{\infty} q^{n} f\left(q^{n} x+\left(1-q^{n}\right) a\right)
$$

for $x \in J$. Moreover, if $c \in(a, x)$ then the definite $q$-integral on $J$ is defined by

$$
\begin{aligned}
\int_{c}^{x} f(t)_{a} d_{q} t & =\int_{a}^{x} f(t)_{a} d_{q} t-\int_{a}^{c} f(t)_{a} d_{q} t \\
& =(1-q)(x-a) \sum_{n=0}^{\infty} q^{n} f\left(q^{n} x+\left(1-q^{n}\right) a\right)-(1-q)(c-a) \sum_{n=0}^{\infty} q^{n} f\left(q^{n} c+\left(1-q^{n}\right) a\right) .
\end{aligned}
$$

Note that if $a=0$, then we have the classical q-integral, which is defined by

$$
\int_{0}^{x} f(t){ }_{0} d_{q} t=(1-q) x \sum_{n=0}^{\infty} q^{n} f\left(q^{n} x\right)
$$

for $x \in[0,+\infty)$.

Theorem 8. [19] Assume that $f, g: J \rightarrow \mathbb{R}$ are continuous functions, $\alpha \in \mathbb{R}$. Then, for $x \in J$,

$$
\begin{aligned}
& \int_{a}^{x}[f(t)+g(t)]_{a} d_{q} t=\int_{a}^{x} f(t)_{a} d_{q} t+\int_{a}^{x} g(t)_{a} d_{q} t \\
& \int_{a}^{x}(\alpha f)(t)_{a} d_{q} t=\alpha \int_{a}^{x} f(t)_{a} d_{q} t .
\end{aligned}
$$

In addition, we introduce the $q$-analogues of $a$ and $(x-a)^{n}$ and the definition of $q$-Beta function.

Definition 4. [22] For any real number a,

$$
[a]_{q}=\frac{q^{a}-1}{q-1}
$$

is called the q-analogue of a. In particular, if $n \in \mathbb{Z}^{+}$, we denote

$$
[n]=\frac{q^{n}-1}{q-1}=q^{n-1}+\cdots+q+1 .
$$

Definition 5. [22] If $n$ is an integer, the q-analogue of $(x-a)^{n}$ is the polynomial

$$
(x-a)_{q}^{n}= \begin{cases}1, & \text { if } n=0, \\ (x-a)(x-q a) \cdots\left(x-q^{n-1} a\right), & \text { if } n \geq 1 .\end{cases}
$$

Definition 6. [22] For any $t, s>0$,

$$
\beta_{q}(t, s)=\int_{0}^{1} x^{t-1}(1-q x)_{q}^{s-1}{ }_{0} d_{q} x
$$

is called the q-Beta function. Note that

$$
\beta_{q}(t, 1)=\int_{0}^{1} x^{t-1}{ }_{0} d_{q} x=\frac{1}{[t]},
$$

where $[t]$ is the q-analogue of $t$. 
At last, we present four simple calculations that will be used in this paper.

Lemma 3. Let $f(x)=1$, then we have

$$
\int_{0}^{1}{ }_{0} d_{q} x=(1-q) \sum_{n=0}^{\infty} q^{n}=1
$$

Lemma 4. Let $f(x)=x$ for $x \in[a, b]$, then we have

$$
\int_{0}^{1} x_{0} d_{q} x=(1-q) \sum_{n=0}^{\infty} q^{2 n}=\frac{1}{1+q} .
$$

Lemma 5. Let $f(x)=1-q x$ for $x \in[0,1]$ where $0<q<1$ be a constant, then we have

$$
\int_{0}^{1}(1-q x)_{0} d_{q} x=\int_{0}^{1}{ }_{0} d_{q} x-q \int_{0}^{1} x_{0} d_{q} x=\frac{1}{1+q} .
$$

Lemma 6. Let $f(x)=x(1-q x)$ for $x \in[0,1]$ where $0<q<1$ be a constant, then we have

$$
\begin{aligned}
\int_{0}^{1} x(1-q x)_{0} d_{q} x & =\int_{0}^{1}\left(x-q x^{2}\right)_{0} d_{q} x=\int_{0}^{1} x_{0} d_{q} x-q \int_{0}^{1} x^{2}{ }_{0} d_{q} x \\
& =\frac{1}{1+q}-q(1-q) \sum_{n=0}^{\infty} q^{3 n}=\frac{1}{1+q}-q \frac{1}{1+q+q^{2}} \\
& =\frac{1}{(1+q)\left(1+q+q^{2}\right)} .
\end{aligned}
$$

In [6], we can find the notion of quasi-convex functions generalizes the notion of convex functions. More exactly, a function $f:[a, b] \rightarrow \mathbb{R}$ is said to be quasi-convex on $[a, b]$ if

$$
f((1-\lambda) x+\lambda y) \leq \sup \{f(x), f(y)\}
$$

holds for any $x, y \in[a, b]$ and $\lambda \in[0,1]$. It's obviously that any convex function is a quasi-convex function. Furthermore, there exist quasi-convex functions which are not convex.

In [11], we have established the following $q$-integral identity and used it to prove some quantum estimates of Hermite-Hadamard type inequalities for convex functions.

Lemma 7. ([11], Lemma 4.1) Let $f: I=[a, b] \subset \mathbb{R} \rightarrow \mathbb{R}$ be a twice q-differentiable function on $I^{0}$ with ${ }_{a} D_{q}^{2} f$ be continuous and integrable on I where $0<q<1$. Then the following identity holds:

$$
\frac{q f(a)+f(b)}{1+q}-\frac{1}{b-a} \int_{a}^{b} f(x)_{a} d_{q} x=\frac{q^{2}(b-a)^{2}}{1+q} \int_{0}^{1} t(1-q t)_{a} D_{q}^{2} f((1-t) a+t b)_{0} d_{q} t .
$$

Remark 1. If $q \rightarrow 1$ and substitute $(1-t) a+t b$ for $s a+(1-s) b$, then (16) reduces to identity (1) in Lemma 1.

\section{Hermite-Hadamard Inequalities for Quasi-Convex Functions}

In this section, we will give some estimates for the left-hand side of the result of (16) through quasi-convex functions. 
Theorem 9. Let $f: I=[a, b] \subset \mathbb{R} \rightarrow \mathbb{R}$ be a twice $q$-differentiable function on $I^{0}$ with ${ }_{a} D_{q}^{2} f$ be continuous and integrable on I where $0<q<1$. If $\left|{ }_{a} D_{q}^{2} f\right|^{r}$ is quasi-convex on $[a, b]$ for $r \geq 1$, then the following inequality holds:

$$
\begin{aligned}
& \left|\frac{q f(a)+f(b)}{1+q}-\frac{1}{b-a} \int_{a}^{b} f(x)_{a} d_{q} x\right| \\
\leq & \frac{q^{2}(b-a)^{2}}{1+q}\left(\frac{1}{1+q}\right)^{1-\frac{1}{r}}\left(h_{1} \sup \left\{\left|{ }_{a} D_{q}^{2} f(a)\right|^{r},\left|{ }_{a} D_{q}^{2} f(b)\right|^{r}\right\}\right)^{\frac{1}{r}},
\end{aligned}
$$

where

$$
h_{1}=(1-q) \sum_{n=0}^{\infty} q^{2 n}\left(1-q^{n+1}\right)^{r}
$$

Proof. Using Lemma 7, Hölder's inequality and the fact that $\left|{ }_{a} D_{q}^{2} f\right|^{r}$ is a quasi-convex function, we have

$$
\begin{aligned}
& \left|\frac{q f(a)+f(b)}{1+q}-\frac{1}{b-a} \int_{a}^{b} f(x)_{a} d_{q} x\right| \\
= & \left|\frac{q^{2}(b-a)^{2}}{1+q} \int_{0}^{1} t(1-q t)_{a} D_{q}^{2} f((1-t) a+t b)_{0} d_{q} t\right| \\
\leq & \frac{q^{2}(b-a)^{2}}{1+q} \int_{0}^{1} t(1-q t)\left|{ }_{a} D_{q}^{2} f((1-t) a+t b)\right|_{0} d_{q} t \\
\leq & \frac{q^{2}(b-a)^{2}}{1+q}\left(\int_{0}^{1} t_{0} d_{q} t\right)^{1-\frac{1}{r}}\left(\int_{0}^{1} t(1-q t)^{r}\left|{ }_{a} D_{q}^{2} f((1-t) a+t b)\right|^{r}{ }_{0} d_{q} t\right)^{\frac{1}{r}} \\
\leq & \frac{q^{2}(b-a)^{2}}{1+q}\left(\int_{0}^{1} t_{0} d_{q} t\right)^{1-\frac{1}{r}}\left(\sup \left\{\left|{ }_{a} D_{q}^{2} f(a)\right|^{r},\left|{ }_{a} D_{q}^{2} f(b)\right|^{r}\right\} \int_{0}^{1} t(1-q t)^{r}{ }_{0} d_{q} t\right)^{\frac{1}{r}}
\end{aligned}
$$

Applying Lemma 4, we have

$$
\begin{aligned}
& \left|\frac{q f(a)+f(b)}{1+q}-\frac{1}{b-a} \int_{a}^{b} f(x)_{a} d_{q} x\right| \\
\leq & \frac{q^{2}(b-a)^{2}}{1+q}\left(\frac{1}{1+q}\right)^{1-\frac{1}{r}}\left(\sup \left\{\left|{ }_{a} D_{q}^{2} f(a)\right|^{r},\left|{ }_{a} D_{q}^{2} f(b)\right|^{r}\right\} \int_{0}^{1} t(1-q t)_{0}^{r} d_{q} t\right)^{\frac{1}{r}} \\
= & \frac{q^{2}(b-a)^{2}}{1+q}\left(\frac{1}{1+q}\right)^{1-\frac{1}{r}}\left(h_{1} \sup \left\{\left|{ }_{a} D_{q}^{2} f(a)\right|^{r},\left|{ }_{a} D_{q}^{2} f(b)\right|^{r}\right\}\right)^{\frac{1}{r}} .
\end{aligned}
$$

It is easy to check that

$$
h_{1}=\int_{0}^{1} t(1-q t)^{r} d_{q} t=(1-q) \sum_{n=0}^{\infty} q^{2 n}\left(1-q^{n+1}\right)^{r}
$$

thus, we get (17).

Remark 2. If $q \rightarrow 1$, then

$$
h_{1}=\int_{0}^{1} t(1-t)^{r} d t=\frac{1}{(r+1)(r+2)}
$$


Inequality (17) reduces to inequality (2) in Theorem 1 due to the fact that

$$
\begin{aligned}
& \frac{(b-a)^{2}}{2}\left(\frac{1}{2}\right)^{1-\frac{1}{r}}\left(\frac{1}{(r+1)(r+2)}\right)^{\frac{1}{r}}\left(\sup \left\{\left|f^{\prime \prime}(a)\right|^{r},\left|f^{\prime \prime}(b)\right|^{r}\right\}\right)^{\frac{1}{r}} \\
= & \frac{(b-a)^{2}}{4}\left(\frac{2}{(r+1)(r+2)}\right)^{\frac{1}{r}}\left(\sup \left\{\left|f^{\prime \prime}(a)\right|^{r},\left|f^{\prime \prime}(b)\right|^{r}\right\}\right)^{\frac{1}{r}} .
\end{aligned}
$$

Corollary 1. In Theorem 9 , if $r$ is a positive integer, then

$$
(1-q t)^{r} \leq(1-q t)^{r},
$$

and (17) reduces to

$$
\begin{aligned}
& \left|\frac{q f(a)+f(b)}{1+q}-\frac{1}{b-a} \int_{a}^{b} f(x)_{a} d_{q} x\right| \\
\leq & \frac{q^{2}(b-a)^{2}}{1+q}\left(\frac{1}{1+q}\right)^{1-\frac{1}{r}}\left(\beta_{q}(2, r+1) \sup \left\{\left|{ }_{a} D_{q}^{2} f(a)\right|^{r},\left|{ }_{a} D_{q}^{2} f(b)\right|^{r}\right\}\right)^{\frac{1}{r}} .
\end{aligned}
$$

Theorem 10. Let $f: I=[a, b] \subset \mathbb{R} \rightarrow \mathbb{R}$ be a twice q-differentiable function on $I^{0}$ with ${ }_{a} D_{q}^{2} f$ be continuous and integrable on I where $0<q<1$. If $\left|{ }_{a} D_{q}^{2} f\right|^{r}$ is quasi-convex on $[a, b]$ where $p, r>1, \frac{1}{p}+\frac{1}{r}=1$, then

$$
\left|\frac{q f(a)+f(b)}{1+q}-\frac{1}{b-a} \int_{a}^{b} f(x)_{a} d_{q} x\right| \leq \frac{q^{2}(b-a)^{2}}{1+q}\left(l_{1}\right)^{\frac{1}{p}}\left(\frac{\sup \left\{\left|{ }_{a} D_{q}^{2} f(a)\right|^{r},\left|{ }_{a} D_{q}^{2} f(b)\right|^{r}\right\}}{1+q}\right)^{\frac{1}{r}}
$$

where

$$
l_{1}=(1-q) \sum_{n=0}^{\infty} q^{2 n}\left(1-q^{n+1}\right)^{p}
$$

Proof. Using Lemma 7, Hölder's inequality and the fact that $\left|{ }_{a} D_{q}^{2} f\right|^{r}$ is a quasi-convex function, we have

$$
\begin{aligned}
& \left|\frac{q f(a)+f(b)}{1+q}-\frac{1}{b-a} \int_{a}^{b} f(x)_{a} d_{q} x\right| \\
= & \left|\frac{q^{2}(b-a)^{2}}{1+q} \int_{0}^{1} t(1-q t)_{a} D_{q}^{2} f((1-t) a+t b)_{0} d_{q} t\right| \\
\leq & \frac{q^{2}(b-a)^{2}}{1+q} \int_{0}^{1} t(1-q t)\left|{ }_{a} D_{q}^{2} f((1-t) a+t b)\right|_{0} d_{q} t \\
\leq & \frac{q^{2}(b-a)^{2}}{1+q}\left(\int_{0}^{1} t(1-q t)^{p}{ }_{0} d_{q} t\right)^{\frac{1}{p}}\left(\int_{0}^{1} t\left|{ }_{a} D_{q}^{2} f((1-t) a+t b)\right|^{r}{ }_{0} d_{q} t\right)^{\frac{1}{r}} \\
\leq & \frac{q^{2}(b-a)^{2}}{1+q}\left(\int_{0}^{1} t(1-q t)^{p}{ }_{0} d_{q} t\right)^{\frac{1}{p}}\left(\sup \left\{\left.\left.\right|_{a} D_{q}^{2} f(a)\right|^{r},\left|a_{a} D_{q}^{2} f(b)\right|^{r}\right\} \int_{0}^{1} t_{0} d_{q} t\right)^{\frac{1}{r}}
\end{aligned}
$$


Applying Lemma 4, we have

$$
\begin{aligned}
& \left|\frac{q f(a)+f(b)}{1+q}-\frac{1}{b-a} \int_{a}^{b} f(x)_{a} d_{q} x\right| \\
\leq & \frac{q^{2}(b-a)^{2}}{1+q}\left(\int_{0}^{1} t(1-q t)^{p}{ }_{0} d_{q} t\right)^{\frac{1}{p}}\left(\frac{\sup \left\{\left|{ }_{a} D_{q}^{2} f(a)\right|^{r},\left|{ }_{a} D_{q}^{2} f(b)\right|^{r}\right\}}{1+q}\right)^{\frac{1}{r}} \\
= & \frac{q^{2}(b-a)^{2}}{1+q}\left(l_{1}\right)^{\frac{1}{p}}\left(\frac{\sup \left\{\left|a_{a} D_{q}^{2} f(a)\right|^{r},\left|{ }_{a} D_{q}^{2} f(b)\right|^{r}\right\}}{1+q}\right)^{\frac{1}{r}} .
\end{aligned}
$$

It is easy to check that

$$
l_{1}=\int_{0}^{1} t(1-q t)^{p} d_{q} t=(1-q) \sum_{n=0}^{\infty} q^{2 n}\left(1-q^{n+1}\right)^{p}
$$

thus, we get (18).

Remark 3. If $q \rightarrow 1$, then

$$
l_{1}=\int_{0}^{1} t(1-t)^{p} d t=\beta(2, p+1) .
$$

Inequality (18) reduces to inequality (3) in Theorem 2.

Corollary 2. In Theorem 10, if $p$ is a positive integer and $p>1$, then

$$
(1-q t)^{p} \leq(1-q t)_{q}^{p}
$$

and (18) reduces to

$$
\left|\frac{q f(a)+f(b)}{1+q}-\frac{1}{b-a} \int_{a}^{b} f(x)_{a} d_{q} x\right| \leq \frac{q^{2}(b-a)^{2}}{1+q}\left(\beta_{q}(2, p+1)\right)^{\frac{1}{p}}\left(\frac{\sup \left\{\left|{ }_{a} D_{q}^{2} f(a)\right|^{r},\left|{ }_{a} D_{q}^{2} f(b)\right|^{r}\right\}}{1+q}\right)^{\frac{1}{r}} .
$$

Theorem 11. Let $f: I=[a, b] \subset \mathbb{R} \rightarrow \mathbb{R}$ be a twice $q$-differentiable function on $I^{0}$ with ${ }_{a} D_{q}^{2} f$ be continuous and integrable on I where $0<q<1$. If $\left|a D_{q}^{2} f\right|^{r}$ is quasi-convex on $[a, b]$ where $p, r>1, \frac{1}{p}+\frac{1}{r}=1$, then the following inequality holds:

$$
\left|\frac{q f(a)+f(b)}{1+q}-\frac{1}{b-a} \int_{a}^{b} f(x)_{a} d_{q} x\right| \leq \frac{q^{2}(b-a)^{2}}{1+q}\left(s_{1}\right)^{\frac{1}{p}}\left(\sup \left\{\left|{ }_{a} D_{q}^{2} f(a)\right|^{r},\left|{ }_{a} D_{q}^{2} f(b)\right|^{r}\right\}\right)^{\frac{1}{r}},
$$

where

$$
s_{1}=(1-q) \sum_{n=0}^{\infty}\left(q^{n}\right)^{p+1}\left(1-q^{n+1}\right)^{p} .
$$


Proof. Using Lemma 7, Hölder's inequality and the fact that $\left|{ }_{a} D_{q}^{2} f\right|^{r}$ is a quasi-convex function, we have

$$
\begin{aligned}
& \left|\frac{q f(a)+f(b)}{1+q}-\frac{1}{b-a} \int_{a}^{b} f(x)_{a} d_{q} x\right| \\
= & \left|\frac{q^{2}(b-a)^{2}}{1+q} \int_{0}^{1} t(1-q t)_{a} D_{q}^{2} f((1-t) a+t b)_{0} d_{q} t\right| \\
\leq & \left.\left.\frac{q^{2}(b-a)^{2}}{1+q} \int_{0}^{1} t(1-q t)\right|_{a} D_{q}^{2} f((1-t) a+t b)\right|_{0} d_{q} t \\
\leq & \frac{q^{2}(b-a)^{2}}{1+q}\left(\int_{0}^{1} t^{p}(1-q t)^{p} d_{q} t\right)^{\frac{1}{p}}\left(\int_{0}^{1}\left|{ }_{a} D_{q}^{2} f((1-t) a+t b)\right|^{r} d_{q} t\right)^{\frac{1}{r}} \\
\leq & \frac{q^{2}(b-a)^{2}}{1+q}\left(\int_{0}^{1} t^{p}(1-q t)^{p} d_{q} t\right)^{\frac{1}{p}}\left(\sup \left\{\left|{ }_{a} D_{q}^{2} f(a)\right|^{r},\left|{ }_{a} D_{q}^{2} f(b)\right|^{r}\right\} \int_{0}^{1}{ }_{0} d_{q} t\right)^{\frac{1}{r}}
\end{aligned}
$$

Applying Lemma 3, we have

$$
\begin{aligned}
& \left|\frac{q f(a)+f(b)}{1+q}-\frac{1}{b-a} \int_{a}^{b} f(x)_{a} d_{q} x\right| \\
\leq & \frac{q^{2}(b-a)^{2}}{1+q}\left(s_{1}\right)^{\frac{1}{p}}\left(\sup \left\{\left.||_{a} D_{q}^{2} f(a)\right|^{r},\left|{ }_{a} D_{q}^{2} f(b)\right|^{r}\right\}\right)^{\frac{1}{r}} .
\end{aligned}
$$

It is easy to check that

$$
s_{1}=\int_{0}^{1} t^{p}(1-q t)^{p} d_{q} t=(1-q) \sum_{n=0}^{\infty}\left(q^{n}\right)^{p+1}\left(1-q^{n+1}\right)^{p},
$$

thus, we get (19).

Remark 4. If $q \rightarrow 1$, then

$$
s_{1}=\int_{0}^{1} t^{p}(1-t)^{p} d t=\beta(p+1, p+1) .
$$

Using the properties of Beta function, that is, $\beta(x, x)=2^{1-2 x} \beta\left(\frac{1}{2}, x\right)$ and $\beta(x, y)=\frac{\Gamma(x) \Gamma(y)}{\Gamma(x y)}$, we can obtain that

$$
\beta(p+1, p+1)=2^{1-2(p+1)} \beta\left(\frac{1}{2}, p+1\right)=2^{-2 p-1} \frac{\Gamma\left(\frac{1}{2}\right) \Gamma(p+1)}{\Gamma\left(\frac{3}{2}+p\right)},
$$

where $\Gamma\left(\frac{1}{2}\right)=\sqrt{\pi}$ and $\Gamma(t)$ is Gamma function:

$$
\Gamma(t)=\int_{0}^{\infty} x^{t-1} e^{-x} d x, \quad t>0 .
$$

Inequality (19) reduces to inequality (5) in Theorem 4 due to the fact that

$$
\begin{aligned}
& \frac{(b-a)^{2}}{2}\left(2^{-2 p-1} \frac{\Gamma\left(\frac{1}{2}\right) \Gamma(p+1)}{\Gamma\left(\frac{3}{2}+p\right)}\right)^{\frac{1}{p}}\left(\sup \left\{\left|f^{\prime \prime}(a)\right|^{r},\left|f^{\prime \prime}(b)\right|^{r}\right\}\right)^{\frac{1}{r}} \\
= & \frac{(b-a)^{2}}{8}\left(\frac{\sqrt{\pi}}{2}\right)^{\frac{1}{p}}\left(\frac{\Gamma(1+p)}{\Gamma\left(\frac{3}{2}+p\right)}\right)^{\frac{1}{p}}\left(\sup \left\{\left|f^{\prime \prime}(a)\right|^{r},\left|f^{\prime \prime}(b)\right|^{r}\right\}\right)^{\frac{1}{r}} .
\end{aligned}
$$


Corollary 3. In Theorem 11, if $p$ is a positive integer, $p>1$, then

$$
(1-q t)^{p} \leq(1-q t)_{q}^{p}
$$

and (19) reduces to

$$
\left|\frac{q f(a)+f(b)}{1+q}-\frac{1}{b-a} \int_{a}^{b} f(x)_{a} d_{q} x\right| \leq \frac{q^{2}(b-a)^{2}}{1+q}\left(\beta_{q}(p+1, p+1)\right)^{\frac{1}{p}}\left(\sup \left\{\left|{ }_{a} D_{q}^{2} f(a)\right|^{r},\left|{ }_{a} D_{q}^{2} f(b)\right|^{r}\right\}\right)^{\frac{1}{r}} .
$$

Theorem 12. Let $f: I=[a, b] \subset \mathbb{R} \rightarrow \mathbb{R}$ be a twice $q$-differentiable function on $I^{0}$ with ${ }_{a} D_{q}^{2} f$ be continuous and integrable on I where $0<q<1$. If $\left|a D_{q}^{2} f\right|^{r}$ is quasi-convex on $[a, b]$ where $p, r>1, \frac{1}{p}+\frac{1}{r}=1$, then the following inequality holds:

$$
\begin{aligned}
& \left|\frac{q f(a)+f(b)}{1+q}-\frac{1}{b-a} \int_{a}^{b} f(x)_{a} d_{q} x\right| \\
\leq & \frac{q^{2}(b-a)^{2}}{1+q}\left(\frac{1}{[p+1]}\right)^{\frac{1}{p}}\left(m_{1} \sup \left\{\left|{ }_{a} D_{q}^{2} f(a)\right|^{r},\left|{ }_{a} D_{q}^{2} f(b)\right|^{r}\right\}\right)^{\frac{1}{r}},
\end{aligned}
$$

where

$$
m_{1}=(1-q) \sum_{n=0}^{\infty} q^{n}\left(1-q^{n+1}\right)^{r}
$$

and $[p+1]$ is the $q$-analogue of $p+1$.

Proof. Using Lemma 7, Hölder's inequality and the fact that $\left|{ }_{a} D_{q}^{2} f\right|^{r}$ is a quasi-convex function, we have

$$
\begin{aligned}
& \left|\frac{q f(a)+f(b)}{1+q}-\frac{1}{b-a} \int_{a}^{b} f(x)_{a} d_{q} x\right| \\
= & \left|\frac{q^{2}(b-a)^{2}}{1+q} \int_{0}^{1} t(1-q t)_{a} D_{q}^{2} f((1-t) a+t b)_{0} d_{q} t\right| \\
\leq & \frac{q^{2}(b-a)^{2}}{1+q} \int_{0}^{1} t(1-q t)\left|{ }_{a} D_{q}^{2} f((1-t) a+t b)\right|_{0} d_{q} t \\
\leq & \frac{q^{2}(b-a)^{2}}{1+q}\left(\int_{0}^{1} t^{p}{ }_{0} d_{q} t\right)^{\frac{1}{p}}\left(\int_{0}^{1}(1-q t)^{r}\left|{ }_{a} D_{q}^{2} f((1-t) a+t b)\right|_{0}^{r} d_{q} t\right)^{\frac{1}{r}} \\
\leq & \frac{q^{2}(b-a)^{2}}{1+q}\left(\int_{0}^{1} t^{p}{ }_{0} d_{q} t\right)^{\frac{1}{p}}\left(\sup \left\{\left|{ }_{a} D_{q}^{2} f(a)\right|^{r},\left|{ }_{a} D_{q}^{2} f(b)\right|^{r}\right\} \int_{0}^{1}(1-q t)^{r} d_{q} t\right)^{\frac{1}{r}}
\end{aligned}
$$

Applying (14) in Definition 6, we have

$$
\begin{aligned}
& \left|\frac{q f(a)+f(b)}{1+q}-\frac{1}{b-a} \int_{a}^{b} f(x)_{a} d_{q} x\right| \\
\leq & \frac{q^{2}(b-a)^{2}}{1+q}\left(\frac{1}{[p+1]}\right)^{\frac{1}{p}}\left(\sup \left\{\left|{ }_{a} D_{q}^{2} f(a)\right|^{r},\left|{ }_{a} D_{q}^{2} f(b)\right|^{r}\right\} \int_{0}^{1}(1-q t)^{r} d_{q} t\right)^{\frac{1}{r}} \\
= & \frac{q^{2}(b-a)^{2}}{1+q}\left(\frac{1}{[p+1]}\right)^{\frac{1}{p}}\left(m_{1} \sup \left\{\left|{ }_{a} D_{q}^{2} f(a)\right|^{r},\left|{ }_{a} D_{q}^{2} f(b)\right|^{r}\right\}\right)^{\frac{1}{r}} .
\end{aligned}
$$

It is easy to check that

$$
m_{1}=\int_{0}^{1}(1-q t)^{r}{ }_{0} d_{q} t=(1-q) \sum_{n=0}^{\infty} q^{n}\left(1-q^{n+1}\right)^{r}
$$


thus, we get (20).

Remark 5. If $q \rightarrow 1$, then

$$
m_{1}=\int_{0}^{1}(1-t)^{r} d t=\frac{1}{r+1}
$$

and (20) reduces to

$$
\left|\frac{f(a)+f(b)}{2}-\frac{1}{b-a} \int_{a}^{b} f(x) d x\right| \leq \frac{(b-a)^{2}}{2}\left(\frac{1}{p+1}\right)^{\frac{1}{p}}\left(\frac{\sup \left\{\left|f^{\prime \prime}(a)\right|^{r},\left|f^{\prime \prime}(b)\right|^{r}\right\}}{r+1}\right)^{\frac{1}{r}} .
$$

Corollary 4. In Theorem 12, if $r$ is a positive integer, $r>1$, then

$$
(1-q t)^{r} \leq(1-q t)_{q^{\prime}}^{r}
$$

and (20) reduces to

$$
\begin{aligned}
& \left|\frac{q f(a)+f(b)}{1+q}-\frac{1}{b-a} \int_{a}^{b} f(x)_{a} d_{q} x\right| \\
\leq & \frac{q^{2}(b-a)^{2}}{1+q}\left(\frac{1}{[p+1]}\right)^{\frac{1}{p}}\left(\beta_{q}(1, r+1) \sup \left\{\left|{ }_{a} D_{q}^{2} f(a)\right|^{r},\left|{ }_{a} D_{q}^{2} f(b)\right|^{r}\right\}\right)^{\frac{1}{r}} .
\end{aligned}
$$

Theorem 13. Let $f: I=[a, b] \subset \mathbb{R} \rightarrow \mathbb{R}$ be a twice q-differentiable function on $I^{0}$ with ${ }_{a} D_{q}^{2} f$ be continuous and integrable on I where $0<q<1$. If $\left|{ }_{a} D_{q}^{2} f\right|^{r}$ is quasi-convex on $[a, b]$ where $p, r>1, \frac{1}{p}+\frac{1}{r}=1$, then the following inequality holds:

$$
\begin{aligned}
& \left|\frac{q f(a)+f(b)}{1+q}-\frac{1}{b-a} \int_{a}^{b} f(x)_{a} d_{q} x\right| \\
\leq & \frac{q^{2}(b-a)^{2}}{1+q}\left(n_{1}\right)^{\frac{1}{p}}\left(\frac{\sup \left\{\left|a D_{q}^{2} f(a)\right|^{r},\left|{ }_{a} D_{q}^{2} f(b)\right|^{r}\right\}}{[r+1]}\right)^{\frac{1}{r}},
\end{aligned}
$$

where

$$
n_{1}=(1-q) \sum_{n=0}^{\infty} q^{n}\left(1-q^{n+1}\right)^{p}
$$

and $[r+1]$ is the $q$-analogue of $r+1$.

Proof. Using Lemma 7, Hölder's inequality and the fact that $\left|{ }_{a} D_{q}^{2} f\right|^{r}$ is a quasi-convex function, we have

$$
\begin{aligned}
& \left|\frac{q f(a)+f(b)}{1+q}-\frac{1}{b-a} \int_{a}^{b} f(x)_{a} d_{q} x\right| \\
= & \left|\frac{q^{2}(b-a)^{2}}{1+q} \int_{0}^{1} t(1-q t)_{a} D_{q}^{2} f((1-t) a+t b)_{0} d_{q} t\right| \\
\leq & \frac{q^{2}(b-a)^{2}}{1+q} \int_{0}^{1} t(1-q t)\left|{ }_{a} D_{q}^{2} f((1-t) a+t b)\right|{ }_{0} d_{q} t \\
\leq & \frac{q^{2}(b-a)^{2}}{1+q}\left(\int_{0}^{1}(1-q t)^{p}{ }_{0} d_{q} t\right)^{\frac{1}{p}}\left(\int_{0}^{1} t^{r}\left|{ }_{a} D_{q}^{2} f((1-t) a+t b)\right|^{r} d_{q} t\right)^{\frac{1}{r}} \\
\leq & \frac{q^{2}(b-a)^{2}}{1+q}\left(\int_{0}^{1}(1-q t)^{p}{ }_{0} d_{q} t\right)^{\frac{1}{p}}\left(\sup \left\{\left|{ }_{a} D_{q}^{2} f(a)\right|^{r},\left|{ }_{a} D_{q}^{2} f(b)\right|^{r}\right\} \int_{0}^{1} t_{0}^{r} d_{q} t\right)^{\frac{1}{r}}
\end{aligned}
$$


Applying (14) in Definition 6, we have

$$
\begin{aligned}
& \left|\frac{q f(a)+f(b)}{1+q}-\frac{1}{b-a} \int_{a}^{b} f(x)_{a} d_{q} x\right| \\
\leq & \frac{q^{2}(b-a)^{2}}{1+q}\left(\int_{0}^{1}(1-q t)^{p}{ }_{0} d_{q} t\right)^{\frac{1}{p}}\left(\frac{\sup \left\{\left|{ }_{a} D_{q}^{2} f(a)\right|^{r},\left|{ }_{a} D_{q}^{2} f(b)\right|^{r}\right\}}{[r+1]}\right)^{\frac{1}{r}} \\
= & \frac{q^{2}(b-a)^{2}}{1+q}\left(n_{1}\right)^{\frac{1}{p}}\left(\frac{\sup \left\{\left|{ }_{a} D_{q}^{2} f(a)\right|^{r},\left|{ }_{a} D_{q}^{2} f(b)\right|^{r}\right\}}{[r+1]}\right)^{\frac{1}{r}} .
\end{aligned}
$$

It is easy to check that

$$
n_{1}=\int_{0}^{1}(1-q t)^{p}{ }_{0} d_{q} t=(1-q) \sum_{n=0}^{\infty} q^{n}\left(1-q^{n+1}\right)^{p}
$$

thus, we get (22).

Remark 6. If $q \rightarrow 1$, then

$$
n_{1}=\int_{0}^{1}(1-t)^{p} d t=\frac{1}{p+1}
$$

and (22) reduces to (21) in Remark 5.

Corollary 5. In Theorem 13, if $p$ is a positive integer, $p>1$, then

$$
(1-q t)^{p} \leq(1-q t)_{q}^{p}
$$

and (22) reduces to

$$
\begin{aligned}
& \left|\frac{q f(a)+f(b)}{1+q}-\frac{1}{b-a} \int_{a}^{b} f(x)_{a} d_{q} x\right| \\
\leq & \frac{q^{2}(b-a)^{2}}{1+q}\left(\beta_{q}(1, p+1)\right)^{\frac{1}{p}}\left(\frac{\sup \left\{\left|{ }_{a} D_{q}^{2} f(a)\right|^{r},\left|{ }_{a} D_{q}^{2} f(b)\right|^{r}\right\}}{[r+1]}\right)^{\frac{1}{r}} .
\end{aligned}
$$

Theorem 14. Let $f: I=[a, b] \subset \mathbb{R} \rightarrow \mathbb{R}$ be a twice $q$-differentiable function on $I^{0}$ with ${ }_{a} D_{q}^{2} f$ be continuous and integrable on I where $0<q<1$. If $\left|a D_{q}^{2} f\right|^{r}$ is quasi-convex on $[a, b]$ for $r \geq 1$, then

$$
\left|\frac{q f(a)+f(b)}{1+q}-\frac{1}{b-a} \int_{a}^{b} f(x)_{a} d_{q} x\right| \leq \frac{q^{2}(b-a)^{2}}{1+q}\left(\mu_{1}\right)^{\frac{1}{r}}\left(\sup \left\{\left|{ }_{a} D_{q}^{2} f(a)\right|^{r},\left|{ }_{a} D_{q}^{2} f(b)\right|^{r}\right\}\right)^{\frac{1}{r}},
$$

where

$$
\mu_{1}=(1-q) \sum_{n=0}^{\infty}\left(q^{n}\right)^{r+1}\left(1-q^{n+1}\right)^{r}
$$


Proof. Using Lemma 7, Hölder's inequality and the fact that $\left|{ }_{a} D_{q}^{2} f\right|^{r}$ is a quasi-convex function, we have

$$
\begin{aligned}
& \left|\frac{q f(a)+f(b)}{1+q}-\frac{1}{b-a} \int_{a}^{b} f(x)_{a} d_{q} x\right| \\
= & \left|\frac{q^{2}(b-a)^{2}}{1+q} \int_{0}^{1} t(1-q t)_{a} D_{q}^{2} f((1-t) a+t b)_{0} d_{q} t\right| \\
\leq & \frac{q^{2}(b-a)^{2}}{1+q}\left(\int_{0}^{1}{ }_{0} d_{q} t\right)^{1-\frac{1}{r}}\left(\int_{0}^{1}|t(1-q t)|^{r}\left|{ }_{a} D_{q}^{2} f((1-t) a+t b)\right|_{0}^{r} d_{q} t\right)^{\frac{1}{r}} \\
\leq & \frac{q^{2}(b-a)^{2}}{1+q}\left(\int_{0}^{1}{ }_{0} d_{q} t\right)^{1-\frac{1}{r}}\left(\sup \left\{\left|{ }_{a} D_{q}^{2} f(a)\right|^{r},\left|{ }_{a} D_{q}^{2} f(b)\right|^{r}\right\}\right)^{\frac{1}{r}}\left(\int_{0}^{1}|t(1-q t)|^{r}{ }_{0} d_{q} t\right)^{\frac{1}{r}}
\end{aligned}
$$

Applying Lemma 3, we have

$$
\begin{aligned}
& \left|\frac{q f(a)+f(b)}{1+q}-\frac{1}{b-a} \int_{a}^{b} f(x)_{a} d_{q} x\right| \\
\leq & \frac{q^{2}(b-a)^{2}}{1+q}\left(\sup \left\{\left|{ }_{a} D_{q}^{2} f(a)\right|^{r},\left|{ }_{a} D_{q}^{2} f(b)\right|^{r}\right\}\right)^{\frac{1}{r}}\left(\int_{0}^{1}|t(1-q t)|^{r} d_{q} t\right)^{\frac{1}{r}} \\
= & \frac{q^{2}(b-a)^{2}}{1+q}\left(\sup \left\{\left|{ }_{a} D_{q}^{2} f(a)\right|^{r},\left|{ }_{a} D_{q}^{2} f(b)\right|^{r}\right\}\right)^{\frac{1}{r}}\left(\mu_{1}\right)^{\frac{1}{r}} .
\end{aligned}
$$

It is easy to check that

$$
\mu_{1}=\int_{0}^{1} t^{r}(1-q t)^{r} d_{q} t=(1-q) \sum_{n=0}^{\infty}\left(q^{n}\right)^{r+1}\left(1-q^{n+1}\right)^{r},
$$

thus, we get (23).

Remark 7. If $q \rightarrow 1$, then

$$
\mu_{1}=\int_{0}^{1} t^{r}(1-t)^{r} d_{q} t=\beta(r+1, r+1)
$$

and (23) reduces to

$$
\left|\frac{f(a)+f(b)}{2}-\frac{1}{b-a} \int_{a}^{b} f(x) d x\right| \leq \frac{(b-a)^{2}}{2}(\beta(r+1, r+1))^{\frac{1}{r}}\left(\sup \left\{\left|f^{\prime \prime}(a)\right|^{r},\left|f^{\prime \prime}(b)\right|^{r}\right\}\right)^{\frac{1}{r}} .
$$

Corollary 6. In Theorem 14, if $r$ is a positive integer, then

$$
(1-q t)^{r} \leq(1-q t)_{q}^{r}
$$

and (23) reduces to

$$
\left|\frac{q f(a)+f(b)}{1+q}-\frac{1}{b-a} \int_{a}^{b} f(x)_{a} d_{q} x\right| \leq \frac{q^{2}(b-a)^{2}}{1+q}\left(\beta_{q}(r+1, r+1)\right)^{\frac{1}{r}}\left(\sup \left\{\left|a_{a} D_{q}^{2} f(a)\right|^{r},\left|a_{a} D_{q}^{2} f(b)\right|^{r}\right\}\right)^{\frac{1}{r}} .
$$


Theorem 15. Let $f: I=[a, b] \subset \mathbb{R} \rightarrow \mathbb{R}$ be a twice $q$-differentiable function on $I^{0}$ with ${ }_{a} D_{q}^{2} f$ be continuous and integrable on I where $0<q<1$. If $\left|{ }_{a} D_{q}^{2} f\right|^{r}$ is quasi-convex on $[a, b]$ for $r \geq 1$, then

$$
\begin{aligned}
& \left|\frac{q f(a)+f(b)}{1+q}-\frac{1}{b-a} \int_{a}^{b} f(x)_{a} d_{q} x\right| \\
\leq & \frac{q^{2}(b-a)^{2}}{1+q}\left(\frac{1}{1+q}\right)^{1-\frac{1}{r}}\left(\beta_{q}(r+1,2) \sup \left\{\left|{ }_{a} D_{q}^{2} f(a)\right|^{r},\left|{ }_{a} D_{q}^{2} f(b)\right|^{r}\right\}\right)^{\frac{1}{r}} .
\end{aligned}
$$

Proof. Using Lemma 7, Hölder's inequality and the fact that $\left|{ }_{a} D_{q}^{2} f\right|^{r}$ is a quasi-convex function, we have

$$
\begin{aligned}
& \left|\frac{q f(a)+f(b)}{1+q}-\frac{1}{b-a} \int_{a}^{b} f(x)_{a} d_{q} x\right| \\
= & \left|\frac{q^{2}(b-a)^{2}}{1+q} \int_{0}^{1} t(1-q t)_{a} D_{q}^{2} f((1-t) a+t b)_{0} d_{q} t\right| \\
\leq & \left.\frac{q^{2}(b-a)^{2}}{1+q} \int_{0}^{1} t(1-q t)\right|_{a} D_{q}^{2} f((1-t) a+t b) \mid{ }_{0} d_{q} t \\
\leq & \frac{q^{2}(b-a)^{2}}{1+q}\left(\int_{0}^{1}(1-q t)_{0} d_{q} t\right)^{1-\frac{1}{r}}\left(\left.\left.\int_{0}^{1}(1-q t) t^{r}\right|_{a} D_{q}^{2} f((1-t) a+t b)\right|^{r}\right)^{\frac{1}{r}} \\
\leq & \frac{q^{2}(b-a)^{2}}{1+q}\left(\int_{0}^{1}(1-q t)_{0} d_{q} t\right)^{1-\frac{1}{r}}\left(\sup \left\{\left|{ }_{a} D_{q}^{2} f(a)\right|^{r},\left|{ }_{a} D_{q}^{2} f(b)\right|^{r}\right\} \int_{0}^{1}(1-q t) t_{0}^{r} d_{q} t\right)^{\frac{1}{r}}
\end{aligned}
$$

Applying Lemma 5 and the fact that $(1-q t)=(1-q t)_{q}^{1}$, we have

$$
\begin{aligned}
& \left|\frac{q f(a)+f(b)}{1+q}-\frac{1}{b-a} \int_{a}^{b} f(x)_{a} d_{q} x\right| \\
\leq & \frac{q^{2}(b-a)^{2}}{1+q}\left(\frac{1}{1+q}\right)^{1-\frac{1}{r}}\left(\sup \left\{\left|{ }_{a} D_{q}^{2} f(a)\right|^{r},\left|{ }_{a} D_{q}^{2} f(b)\right|^{r}\right\} \int_{0}^{1} t^{r}(1-q t)_{q 0}^{1} d_{q} t\right)^{\frac{1}{r}} \\
= & \frac{q^{2}(b-a)^{2}}{1+q}\left(\frac{1}{1+q}\right)^{1-\frac{1}{r}}\left(\beta_{q}(r+1,2) \sup \left\{\left|{ }_{a} D_{q}^{2} f(a)\right|^{r},\left|{ }_{a} D_{q}^{2} f(b)\right|^{r}\right\}\right)^{\frac{1}{r}},
\end{aligned}
$$

thus, we gett (24).

Remark 8. If $q \rightarrow 1$, then

$$
\beta(r+1,2)=\int_{0}^{1} t^{r}(1-t)_{0} d_{q} t=\frac{1}{(r+1)(r+2)},
$$

and (24) reduces to inequality (2) in Theorem 1.

Theorem 16. Let $f: I=[a, b] \subset \mathbb{R} \rightarrow \mathbb{R}$ be a twice $q$-differentiable function on $I^{0}$ with ${ }_{a} D_{q}^{2} f$ be continuous and integrable on I where $0<q<1$. If $\left|{ }_{a} D_{q}^{2} f\right|^{r}$ is quasi-convex on $[a, b]$ where $p, r>1, \frac{1}{p}+\frac{1}{r}=1$, then

$$
\begin{aligned}
& \left|\frac{q f(a)+f(b)}{1+q}-\frac{1}{b-a} \int_{a}^{b} f(x)_{a} d_{q} x\right| \\
\leq & \frac{q^{2}(b-a)^{2}}{1+q}\left(\beta_{q}(p+1,2)\right)^{\frac{1}{p}}\left(\frac{\sup \left\{\left|{ }_{a} D_{q}^{2} f(a)\right|^{r},\left.\left|{ }_{a} D_{q}^{2} f(b)\right|\right|^{r}\right\}}{1+q}\right)^{\frac{1}{r}} .
\end{aligned}
$$


Proof. Using Lemma 7, Hölder's inequality and the fact that $\left|{ }_{a} D_{q}^{2} f\right|^{r}$ is a quasi-convex function, we have

$$
\begin{aligned}
& \left|\frac{q f(a)+f(b)}{1+q}-\frac{1}{b-a} \int_{a}^{b} f(x)_{a} d_{q} x\right| \\
= & \left|\frac{q^{2}(b-a)^{2}}{1+q} \int_{0}^{1} t(1-q t)_{a} D_{q}^{2} f((1-t) a+t b)_{0} d_{q} t\right| \\
\leq & \frac{q^{2}(b-a)^{2}}{1+q} \int_{0}^{1} t(1-q t)\left|{ }_{a} D_{q}^{2} f((1-t) a+t b)\right|_{0} d_{q} t \\
\leq & \frac{q^{2}(b-a)^{2}}{1+q}\left(\int_{0}^{1} t^{p}(1-q t)_{0} d_{q} t\right)^{\frac{1}{p}}\left(\int_{0}^{1}(1-q t)\left|{ }_{a} D_{q}^{2} f((1-t) a+t b)\right|_{0}^{r} d_{q} t\right)^{\frac{1}{r}} \\
\leq & \frac{q^{2}(b-a)^{2}}{1+q}\left(\int_{0}^{1} t^{p}(1-q t)_{0} d_{q} t\right)^{\frac{1}{p}}\left(\sup \left\{\left|a_{a} D_{q}^{2} f(a)\right|^{r},\left|{ }_{a} D_{q}^{2} f(b)\right|^{r}\right\} \int_{0}^{1}(1-q t)_{0} d_{q} t\right)^{\frac{1}{r}}
\end{aligned}
$$

Applying Lemma 5 and the fact that $(1-q t)=(1-q t))_{q}^{1}$, we have

$$
\begin{aligned}
& \left|\frac{q f(a)+f(b)}{1+q}-\frac{1}{b-a} \int_{a}^{b} f(x)_{a} d_{q} x\right| \\
\leq & \frac{q^{2}(b-a)^{2}}{1+q}\left(\int_{0}^{1} t^{p}(1-q t)_{q 0}^{1} d_{q} t\right)^{\frac{1}{p}}\left(\frac{\sup \left\{\left|{ }_{a} D_{q}^{2} f(a)\right|^{r},\left|{ }_{a} D_{q}^{2} f(b)\right|^{r}\right\}}{1+q}\right)^{\frac{1}{r}} \\
= & \frac{q^{2}(b-a)^{2}}{1+q}\left(\beta_{q}(p+1,2)\right)^{\frac{1}{p}}\left(\frac{\sup \left\{\left|{ }_{a} D_{q}^{2} f(a)\right|^{r},\left|{ }_{a} D_{q}^{2} f(b)\right|^{r}\right\}}{1+q}\right)^{\frac{1}{r}},
\end{aligned}
$$

thus, we get (25).

Remark 9. If $q \rightarrow 1$, then

$$
\beta(p+1,2)=\int_{0}^{1} t^{p}(1-t) d t=\int_{0}^{1} s(1-s)^{p} d s=\beta(2, p+1) .
$$

Inequality (25) reduces to inequality (3) in Theorem 2.

Theorem 17. Let $f: I=[a, b] \subset \mathbb{R} \rightarrow \mathbb{R}$ be a twice $q$-differentiable function on $I^{0}$ with ${ }_{a} D_{q}^{2} f$ be continuous and integrable on I where $0<q<1$. If $\left|{ }_{a} D_{q}^{2} f\right|$ is quasi-convex on $[a, b]$, then

$$
\left|\frac{q f(a)+f(b)}{1+q}-\frac{1}{b-a} \int_{a}^{b} f(x)_{a} d_{q} x\right| \leq \frac{q^{2}(b-a)^{2} \sup \left\{\left|{ }_{a} D_{q}^{2} f(a)\right|,\left|{ }_{a} D_{q}^{2} f(b)\right|\right\}}{(1+q)^{2}\left(1+q+q^{2}\right)} .
$$


Proof. Using Lemma 7, Hölder's inequality and the fact that $\left|{ }_{a} D_{q}^{2} f\right|$ is a quasi-convex function, we have

$$
\begin{aligned}
& \left|\frac{q f(a)+f(b)}{1+q}-\frac{1}{b-a} \int_{a}^{b} f(x)_{a} d_{q} x\right| \\
= & \left|\frac{q^{2}(b-a)^{2}}{1+q} \int_{0}^{1} t(1-q t)_{a} D_{q}^{2} f((1-t) a+t b)_{0} d_{q} t\right| \\
\leq & \frac{q^{2}(b-a)^{2}}{1+q} \int_{0}^{1} t(1-q t)\left|{ }_{a} D_{q}^{2} f((1-t) a+t b)\right|{ }_{0} d_{q} t \\
\leq & \frac{q^{2}(b-a)^{2}}{1+q} \sup \left\{\left|{ }_{a} D_{q}^{2} f(a)\right|,\left|{ }_{a} D_{q}^{2} f(b)\right|\right\} \int_{0}^{1} t(1-q t)_{0} d_{q} t
\end{aligned}
$$

Applying Lemma 6, we have

$$
\begin{aligned}
& \left|\frac{q f(a)+f(b)}{1+q}-\frac{1}{b-a} \int_{a}^{b} f(x)_{a} d_{q} x\right| \\
\leq & \frac{q^{2}(b-a)^{2} \sup \left\{\left|{ }_{a} D_{q}^{2} f(a)\right|,\left|{ }_{a} D_{q}^{2} f(b)\right|\right\}}{(1+q)^{2}\left(1+q+q^{2}\right)},
\end{aligned}
$$

thus, we get (26).

Remark 10. If $q \rightarrow 1$, then inequality (26) reduces to inequality (4) in Theorem 3.

Theorem 18. Let $f: I=[a, b] \subset \mathbb{R} \rightarrow \mathbb{R}$ be a twice $q$-differentiable function on $I^{0}$ with ${ }_{a} D_{q}^{2} f$ be continuous and integrable on I where $0<q<1$. If $\left|{ }_{a} D_{q}^{2} f\right|^{r}$ is quasi-convex on $[a, b]$ for $r \geq 1$, then the following inequality holds:

$$
\left|\frac{q f(a)+f(b)}{1+q}-\frac{1}{b-a} \int_{a}^{b} f(x)_{a} d_{q} x\right| \leq \frac{q^{2}(b-a)^{2}}{(1+q)^{2}\left(1+q+q^{2}\right)}\left(\sup \left\{\left|a D_{q}^{2} f(a)\right|^{r},\left|{ }_{a} D_{q}^{2} f(b)\right|^{r}\right\}\right)^{\frac{1}{r}} .
$$

Proof. Using Lemma 7, Hölder's inequality and the fact that $\left|{ }_{a} D_{q}^{2} f\right|^{r}$ is a quasi-convex function, we have

$$
\begin{aligned}
& \left|\frac{q f(a)+f(b)}{1+q}-\frac{1}{b-a} \int_{a}^{b} f(x)_{a} d_{q} x\right| \\
= & \left|\frac{q^{2}(b-a)^{2}}{1+q} \int_{0}^{1} t(1-q t)_{a} D_{q}^{2} f((1-t) a+t b)_{0} d_{q} t\right| \\
\leq & \left.\left.\frac{q^{2}(b-a)^{2}}{1+q} \int_{0}^{1} t(1-q t)\right|_{a} D_{q}^{2} f((1-t) a+t b)\right|_{0} d_{q} t \\
\leq & \frac{q^{2}(b-a)^{2}}{1+q}\left(\int_{0}^{1} t(1-q t)_{0} d_{q} t\right)^{1-\frac{1}{r}}\left(\left.\left.\int_{0}^{1} t(1-q t)\right|_{a} D_{q}^{2} f((1-t) a+t b)\right|_{0} ^{r} d_{q} t\right)^{\frac{1}{r}} \\
\leq & \frac{q^{2}(b-a)^{2}}{1+q}\left(\int_{0}^{1} t(1-q t)_{0} d_{q} t\right)^{1-\frac{1}{r}}\left(\sup \left\{\left|{ }_{a} D_{q}^{2} f(a)\right|^{r},\left|{ }_{a} D_{q}^{2} f(b)\right|^{r}\right\} \int_{0}^{1} t(1-q t)_{0} d_{q} t\right)^{\frac{1}{r}}
\end{aligned}
$$


Applying Lemma 6, we have

$$
\begin{aligned}
& \left|\frac{q f(a)+f(b)}{1+q}-\frac{1}{b-a} \int_{a}^{b} f(x)_{a} d_{q} x\right| \\
\leq & \frac{q^{2}(b-a)^{2}}{1+q}\left(\frac{1}{(1+q)\left(1+q+q^{2}\right)}\right)^{1-\frac{1}{r}}\left(\frac{\sup \left\{\left|{ }_{a} D_{q}^{2} f(a)\right|^{r},\left|{ }_{a} D_{q}^{2} f(b)\right|^{r}\right\}}{(1+q)\left(1+q+q^{2}\right)}\right)^{\frac{1}{r}} \\
= & \frac{q^{2}(b-a)^{2}}{(1+q)^{2}\left(1+q+q^{2}\right)}\left(\sup \left\{\left|{ }_{a} D_{q}^{2} f(a)\right|^{r},\left|{ }_{a} D_{q}^{2} f(b)\right|^{r}\right\}\right)^{\frac{1}{r}},
\end{aligned}
$$

thus, we get (27).

Remark 11. If $q \rightarrow 1$, then inequality (27) reduces to inequality (6) in Theorem 5.

\section{Discussion of New Perspectives}

Currently, the Hermite-Hadamard inequality plays a significant role in the development of all fields of Mathematics. It has sgnificant applications in a variety of applied Mathematics, such as integral inequalities, approximation theory, special means theory, optimization theory, information theory and numerical analysis. In recent years, a number of authors have discovered new Hermite-Hadamard-type inequalities for convex, s-convex functions, logarithmic convex functions, $h$-convex functions, quasi-convex functions, $m$-convex functions, $(K, m)$-convex functions, co-ordinated convex functions, and the Godunova-Levin function, $P$-function, and so on. In this paper, we use a new quantum integral identity established in [11] (Lemma 4.1) to develop some quantum estimates for Hermite-Hadamard type inequalities in which some quasi-convex functions are involved.

Since quantum calculus has large applications in many mathematical areas such as number theory, special functions, quantum mechanics and mathematical inequalities, we hope interested readers will continue to explore more quantum estimates of Hermite-Hadamard type inequalities for other kinds of convex functions, and, furthermore, to find applications in the above-mentioned mathematical areas.

Author Contributions: The work presented here was carried out in collaboration between all authors. All authors contributed equally and significantly in writing this article. All authors have read and approved the final manuscript.

Funding: This research was funded by the National Natural Science Foundation of China (11771216), the Natural Science Foundation of Jiangsu Province (BK20151523), the Six Talent Peaks Project in Jiangsu Province (2015-XCL-020), and the Qing Lan Project of Jiangsu Province.

Acknowledgments: The authors thank the referees for their valuable suggestions and remarks.

Conflicts of Interest: The authors declare no conflict of interest.

\section{References}

1. Pečarić, J.E.; Proschan, F.; Tong, Y.L. Convex Functions, Partial Orderings, and Statistical Applications; Mathematics in Science and Engineering, 187; Academic Press, Inc.: Boston, MA, USA, 1992.

2. Alomari, M.; Darus, M.; Dragomir, S.S. New inequalities of Hermite-Hadamard type for functions whose second derivatives absolute values are quasi-convex. Tamkang J. Math. 2010, 41, 353-359.

3. Dragomir, S.S.; Agarwal, R.P. Two inequalities for differentiable mappings and applications to special means of real numbers and to trapezoidal formula. Appl. Math. Lett. 1998, 11, 91-95. [CrossRef]

4. Dragomir, S.S. On some new inequalities of Hermite-Hadamard type for $m$-convex functions. Tamkang J. Math. 2002, 33, 55-65.

5. Dragomir, S.S.; Fitzpatrick, S. The Hadamard inequalities for s-convex functions in the second sense. Demonstratio Math. 1999, 32, 687-696. [CrossRef]

6. Ion, D.A. Some estimates on the Hermite-Hadamard inequality through quasi-convex functions. Ann. Univ. Craiova Ser. Mat. Inform. 2007, 34, 83-88. 
7. Liu, W.J. New integral inequalities via $(\alpha, m)$-convexity and quasi-convexity. Hacet. J. Math. Stat. 2013, 42, 289-297.

8. Liu, W.J. Some Simpson type inequalities for $h$-convex and $(\alpha, m)$-convex functions. J. Comput. Anal. Appl. 2014, 16, 1005-1012.

9. Liu, W.J. Ostrowski type fractional integral inequalities for MT-convex functions. Miskolc Math. Notes 2015, 16, 249-256. [CrossRef]

10. Liu, W.J.; Wen, W.S.; Park, J.K. Hermite-Hadamard type inequalities for MT-convex functions via classical integrals or fractional integrals. J. Nonlinear Sci. Appl. 2016, 9, 766-777. [CrossRef]

11. Liu, W.J.; Zhuang, H.F. Some quantum estimates of Hermite-Hadamard inequalities for convex functions. J. Appl. Anal. Comput. 2017, 7, 501-522.

12. Liu, Z. Generalization and improvement of some Hadamard type inequalities for Lipschitzian mappings. J. Pure Appl. Math. Adv. Appl. 2009, 1, 175-181.

13. Noor, M.A.; Noor, K.I.; Awan, M.U. Some quantum estimates for Hermite-Hadamard inequalities. Appl. Math. Comput. 2015, 251, 675-679. [CrossRef]

14. Noor, M.A.; Noor, K.I.; Awan, M.U. Some quantum integral inequalities via preinvex functions. Appl. Math. Comput. 2015, 269, 242-251. [CrossRef]

15. Noor, M.A.; Noor, K.I.; Awan, M.U. Quantum analogues of Hermite-Hadamard type inequalities for generalized convexity. In Computation, Cryptography and Network Security; Daras, N., Rassias, M.T., Eds.; Springer: Cham, Switzerland, 2015; pp. 413-439.

16. Özdemir, M.E. On Iyengar-type inequalities via quasi-convexity and quasi-concavity. Miskolc Math. Notes 2014, 15, 171-181. [CrossRef]

17. Sarikaya, M.Z.; Set, E.; Özdemir, M.E. On some new inequalities of Hadamard type involving $h$-convex functions. Acta Math. Univ. Comen. (N.S.) 2010, 79, 265-272.

18. Sudsutad, W.; Ntouyas, S.K.; Tariboon, J. Quantum integral inequalities for convex functions. J. Math. Inequal. 2015, 9, 781-793. [CrossRef]

19. Tariboon, J.; Ntouyas, S.K. Quantum integral inequalities on finite intervals. J. Inequal. Appl. 2014, $2014,121$. [CrossRef]

20. Tariboon, J.; Ntouyas, S.K. Quantum calculus on finite intervals and applications to impulsive difference equations. Adv. Differ. Equ. 2013, 2013, 282. [CrossRef]

21. Al-Salam, W.A. q-Bernoulli numbers and polynomials. Math. Nachr. 1959, 17, 239-260. [CrossRef]

22. Kac, V.; Cheung, P. Quantum Calculus; Universitext; Springer: New York, NY, USA, 2002.

23. Von Neumann, J. Mathematical Foundations of Quantum Mechanics, new ed.; translated from the German and with a preface by Robert T. Beyer; Princeton University Press: Princeton, NJ, USA, 2018.

24. Alp, N.; Sarikaya, M.Z.; Kunt, M.; Iscan, I. q-Hermite Hadamard inequalities and quantum estimates for midpoint type inequalities via convex and quasi-convex functions. J. King Saud Univ. Sci. 2018, 30, 193-203. [CrossRef]

(C) 2019 by the authors. Licensee MDPI, Basel, Switzerland. This article is an open access article distributed under the terms and conditions of the Creative Commons Attribution (CC BY) license (http://creativecommons.org/licenses/by/4.0/). 